\title{
DEVELOPMENT OF OPERATING SPEED MODELS FOR DIFFERENT VEHICLE CLASSES ON RURAL TWO-LANE HIGHWAYS
}

\author{
Ibrahim Hassan Hashim \\ Department of Civil Engineering, Faculty of Engineering, Minoufiya University, \\ Shebin El-Kom, Egypt
}

\begin{abstract}
:
The aim of this study was to develop operating speed models for different vehicle classes for rural two-lane highways. Ten horizontal curves, connected with relatively long tangents, with various geometric characteristics, were selected to perform this study. A series of traffic surveys were carried out using automatic traffic counters, to collect sufficient speed data for different vehicle classes. Preliminary analysis confirmed the necessity of the classification of the vehicles in order to develop operating speed models. Further analysis looked at the differences between speeds on inside and outside lanes of curves and significant differences were found between the speeds of the two directions. Several operating speed models were developed for tangents and curves for different vehicle classes. These models are very useful and can be used for design consistency evaluations.

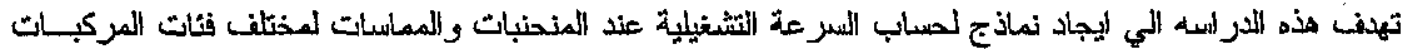

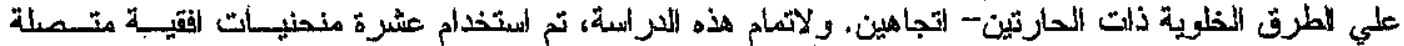

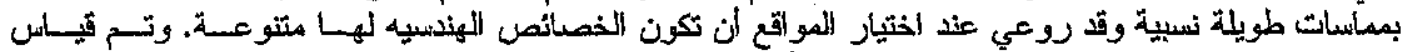

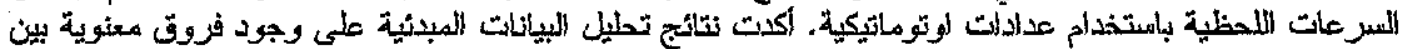

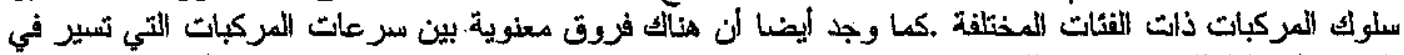

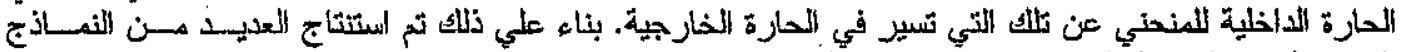

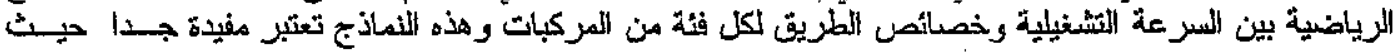

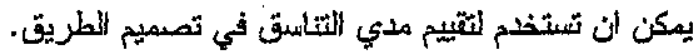

Keywords: Vehicle Classes - Operating Speed - Design Consistency - ANOVA - Regression Analysis
\end{abstract}

\section{INTRODUCTION}

Design consistency is defined as the conformance of the geometric features of a road with driver's expectations. There are several methods to evaluate the consistency of a design in a highway; namely, alignment indices, driver workload, and operating speed based measures [1]. Among these methods, the operating speed approach has been known as the most efficient and quantified measure to date. It is more accurate than the alignment indices method while being much less sophisticated and more quantifiable than the driver workload method. The operating speed is defined as the speed at or below which, the majority of the drivers (e.g., $85 \%$ ) are traveling, while not being impeded by any factors but the geometric features of the road [2].

In the operating speed approach, the consistency of design can be evaluated based on one of two different ways or a combination of both. The first way is evaluating the consistency based on the difference of operating speed between two successive elements of a road (two curves or curve-tangent). The second way, on the other hand, is based on the difference between the operating speed and design speed values. If the operating speed differential between two successive elements or the difference between the operating and design sppeed values is greater than a certain value, the feature is considered inconsistent or the highway design is considered poor [3].

Therefore, the first step that should be followed to evaluate the design consistency is the development of regression models to be used by highway designers/practitioners. These models can predict the operating speed of an element (i.e. curve, tangent); and consequently the speed differential between the two elements or the difference between operating speed and design speed of an element can be calculated. These models should be able to predict the speed values based on the geometric characteristics of the road elements such as curve radius, curve length, deflection angle, etc.

The literature review revealed that many studies have been conducted to develop operating speed models for cars at mid points of horizontal curves for two- 
lane highways [4]. Many factors are found to affect the prediction of operating speed, such as, radius of horizontal curve, length of horizontal curve, deflection angle. Most of them confirmed that a definite relationship exists between operating speed and horizontal curve radius. Generally, when the radius of curve decreases, the operating speed decreases.

Yet, it seems that no research has been carried out to develop operating speed for different vehicle classes (i.e. cars, light good vehicle, heavy good vehicles, and buses). It is likely that operating speeds vary significantly depending on the vehicle type. This could be due to varying vehicle characteristics. As a result, it is important to develop models that are capable of direct calculation of operating speed for different vehicle classes (passenger cars (CAR), Light good Vehicle (LGV), Heavy Good Vehicles (HGV), and Buses (BUS)). Considering the aforementioned statements, this study has three main objectives, as follows:

- Studying the difference of speed behavior between different vehicle classes (CAR, LGV, HGV, and BUS);

- Studying the impact of traffic lane location (inside lane/outside lane) on vehicle speeds in the case of horizontal curves; and

- Development of operating speed models at mid points of horizontal curves and tangents for different vehicle classes.

The scope of this study is limited to two-lane rural highways for dry pavement conditions. The only acceptable traffic condition, used to calculate operating speed, is the free-flow stream of traffic in which no driver is impeded by other vehicles (i.e. headway equals 5 seconds or more). In other words, the focus of this study is on the circumstances in which only the geometric features of a road element are the predominant factors influencing drivers' behavior.

\section{DATA COLLECTION}

One of the most important steps in order to achieve the objectives of this study is to collect the necessary data with a sufficient degree of accuracy. This section provides a detailed description of the data collection process.

\subsection{Geometry Data}

Ten horizontal curves, connected with relatively long tangents, with various geometric characteristics (i.e. curve radius, length), were chosen to perform this study. All of the curves are located in rural two-lane sections with a speed limit of $60 \mathrm{mph}(97 \mathrm{~km} / \mathrm{h})$, from north-east England. Also, all of the chosen sites were located on relatively level terrain to minimize or avoid the effect of the longitudinal gradient. Table (1) shows the geometric characteristics of these sites.

Table (1): Characteristic of the Geometry Data

\begin{tabular}{|l|c|c|c|}
\hline & Max. & Min. & Avg. \\
\hline Curve radius (m) & 747 & 92 & 319 \\
\hline Curve length (m) & 350 & 75 & 181 \\
\hline Deflection angle (deg) & 47 & 21 & 34 \\
\hline Tangent length (m) & 2324 & 185 & 886 \\
\hline
\end{tabular}

\subsection{Speed Data}

There are many techniques that can be used to measure the speed of a vehicle at a specific point on a roadway section. The most well known techniques are: stopwatch, radar guns, and pneumatic traffic counters.

In this study, speed data were collected using automatic pneumatic traffic counters. As they are automatic, a long period of speed survey could practically be carried out. The counters classify traffic by vehicle type and direction of flow. Moreover, the counters are accurate and have the advantage of eliminating the natural tendency of drivers to reduce their speed because of the presence of human observers equipped with radar guns.

The automatic counters made and supplied by MetroCount Company [5] were used, as in figure (1), to perform this study.

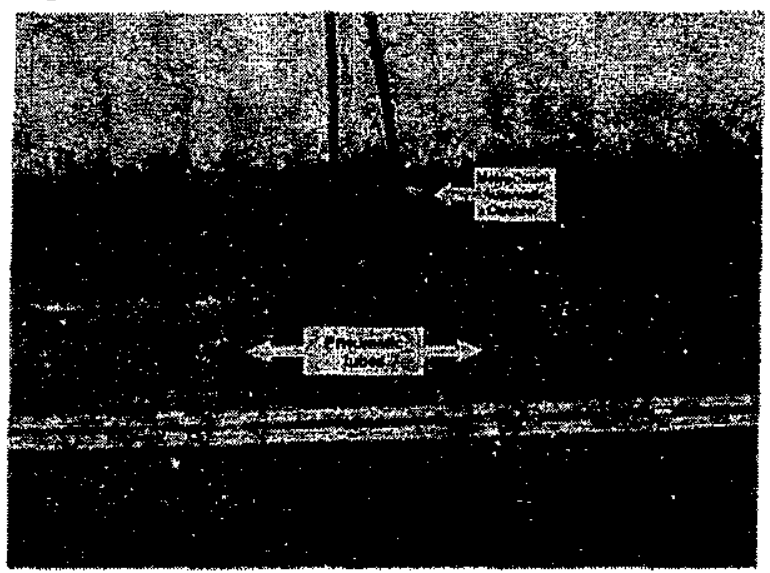

Figure (1) Setting up the Automatic Traffic Counter

In each one of the ten sites (horizontal curves and connecting tangents), the data was collected at five points, as follows:

- The midpoint of the tangent (A);

- The approach point to the horizontal curve (B);

- The start point of the horizontal curve (C);

- The midpoint of the horizontal curve (D); and

- The end point of the horizontal curve (E).

Figure (2) shows the positions of the five counters at each site. 


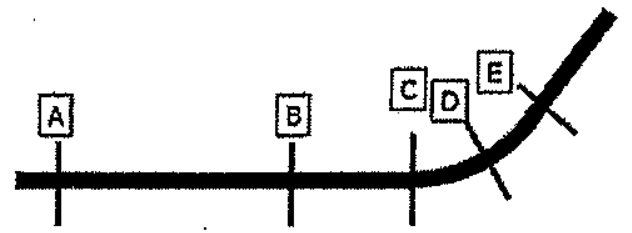

Figure (2): Positions of the Five Counters at Each Site

The counters were left for at least 24 hours at each surveyed site. Subsequently, the stored data was uploaded to the computer using MetroCount software (MetroCount, 2002). Then data analysis was carried out using another component of Metrocount software. This software has the ability to classify the traffic according to direction, vehicle type (using several schemes), headway, time of day and others. In this study, the DFT-UK scheme was used for vehicle classification, as the data were collected from UK roads.

\section{PRELIMINARY ANALYSIS}

The analysis was carried out to compare the speed behavior of vehicles belonging to different classes. SPSS statistical computer program was used to perform the analysis [6]. In this section the ANOVAtest was used to perform this comparison. ANOVA tests the difference between the means of three or more groups, similar to t-test, but t-test tests the difference between the means of two groups.

The vehicle speeds were classified into four classes namely: Cars (CAR); Light Good Vehicles (LGV); Heavy Good Vehicles (HGV); and Buses (BUS). This classification was performed using the MetroCount software and applying a classification scheme called Dft-UK (as the used data was collected from UK roads).

The comparison was performed for each direction of travel $(A B$ or $B A)$, at each point of the five points ( $A$, $B, C, D$, and $E$ ) in each site of the ten sites, resulting in 100 comparisons. Table (2) presents example of the results of 2 of these comparisons. The table shows the results of site 2 , for point $A$ (midpoint of the tangent), for both directions; $A B$ and $B A$. The results showed that the significance values, for $A B$ and $\mathrm{BA}$ are equal to 0.000 (less than 0.05 ) which means that there is a statistical difference between the mean speeds for different vehicle classes, in both directions.

Also, Table (2) confirms these findings, showing that the multiple comparisons of mean differences between vehicle classes are generally significant. The only exception is the difference between HGV and BUS as the significance values are equal to 0.935 and 0.812 (greater than 0.05 ) which means that there is no statistical difference between the mean speeds of HGV and BUS.

Table (2) An Example of ANOVA Results at Site Point A

\begin{tabular}{|c|c|c|c|c|c|c|c|c|}
\hline Sitie & 6010 & DII cection & Significance & 4 & Mutiple & nopa & Hificance) & $4 x^{2}$ \\
\hline \multirow{9}{*}{2} & \multirow[t]{9}{*}{. } & & & & CAR & LGV & HGV & BUS \\
\hline & & \multirow{4}{*}{$\mathbf{A B}$} & \multirow{4}{*}{0.000} & CAR & - & 0.000 & 0.000 & 0.034 \\
\hline & & & & LGV & 0.000 & - & 0.000 & 0.001 \\
\hline & & & & HGV & 0.000 & 0.000 & - & 0.935 \\
\hline & & & & BUS & 0.034 & 0.001 & 0.935 & - \\
\hline & & \multirow{4}{*}{$\mathbf{B A}$} & \multirow{4}{*}{0.000} & CAR & - & 0.001 & 0.000 & 0.031 \\
\hline & & & & LGV & 0.001 & - & 0.000 & 0.004 \\
\hline & & & & HGV & 0.000 & 0.000 & - & 0.812 \\
\hline & & & & BUS & 0.031 & 0.004 & 0.812 & - \\
\hline
\end{tabular}

The results of the 100 comparisons were summarized, and presented in Table 3, as follows:

Table (3) Percent of Significance Differences between Different Vehicle Classes

\begin{tabular}{|c|c|c|c|c|}
\hline 原 & 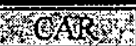 & 160 & YST & STS S \\
\hline 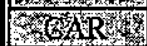 & - & 71 & .92 & 60 \\
\hline 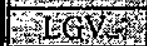 & & - & 94 & 69 \\
\hline r H K & & & - & 11 \\
\hline K & & & & - \\
\hline
\end{tabular}

From this table, it was found that there is a statistically significance difference between the speeds of:
- CAR and LGV in $71 \%$ of the cases.

- CAR \& HGV in $92 \%$ of the cases.

- CAR and BUS in $60 \%$ of the cases.

- LGV and HGV in $94 \%$ of the cases.

- LGV and BUS in $69 \%$ of the cases.

- HGV and BUS in $11 \%$ of the cases.

Therefore, the ANOVA analysis confirmed the necessity of the classification of the vehicles in most of the cases (CAR/LGV, CAR/HGV, CAR/BUS, LGV/HGV, and LGV/BUS). It was also proved that there is no statistically significant difference between the speeds of HGV and BUS, in most of the cases. As 
a result, HGV and BUS could be safely combined together in one class (HV). Consequently, three classes of vehicles (CAR, LGV and HV) will be considered for the purpose of model development.

Another issue that will be addressed here is the influence of the traffic lane location (Inner/Outer) on speed behavior in the case of horizontal curves. A ttest was carried out by looking at the difference in speed distributions for the two lanes of traffic in the same horizontal curve. The analysis was performed, in the 10 curves, for different vehicle classes (CAR, LGV, and HV) as well as for the whole classes as a one dataset. Therefore, the t-test was performed forty times.

The results show that in $100 \%$ of the cases there was a statistically significant difference between the speed distributions of the two lanes of traffic (Inner/Outer). Table (4) shows an example of the results, in the case of CAR for 2 curves, as follows:

Table (4) Impact of Lane of Traffic on Speed Behavior (CAR/Curves 1\&2)

\begin{tabular}{|c|c|c|c|c|c|}
\hline \multicolumn{6}{|c|}{ 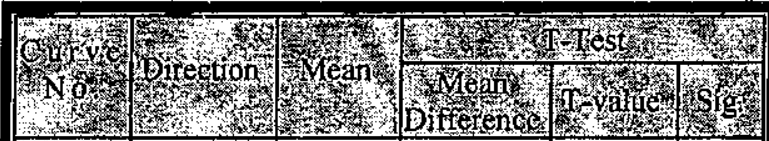 } \\
\hline 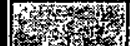 & Inner & 58.54 & \multirow{2}{*}{4.42} & \multirow{2}{*}{17.27} & \multirow{2}{*}{0.000} \\
\hline 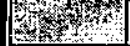 & Outer & 54.12 & & & \\
\hline 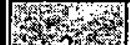 & Inner & 67.39 & \multirow{2}{*}{2.70} & \multirow{2}{*}{8.99} & \multirow{2}{*}{0.000} \\
\hline 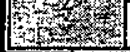 & Outer & 64.69 & & & \\
\hline
\end{tabular}

As a result an independent variable, a dummy variable, for direction of travel will be introduced and considered in the model development stage, in the case of horizontal curves, as in the next section.

\section{OPERATING SPEED MODELS FOR DIFFERENT VEHICLE CLASSES}

In this section, regression analysis was used to establish speed models for the effect of highway geometry (tangent length, curve radius, curve length, deflection angle, and direction of flow) on the operating speed at the midpoints of tangents and curves for different vehicle classes. The analysis used spot speed data to determine the, dependent variable, observed operational $\left(85^{\text {th }}\right.$ percentile) speed.

The analysis considered many mathematical forms of the independent variables (curve radius or the tangent length). These forms are linear, inverse, square root and inverse of square root. The criteria used to assess the predictive accuracy of the models were:

- The coefficient of determination $\left(\mathrm{R}^{2}\right)$ must be as high as possible and significant at the $95 \%$ confidence level. $\mathrm{R}^{2}$ values only give a guide to the "goodness-of-fit"; and

- Each of the independent variables should have regression coefficients that are significantly different from zero, and whose sign should logically explain the effect of this variable on operating speed.

\section{Tangent Models:}

Table (5) summarizes the best tangent models, as follows:

Table (5) Tangent Operating Speed Models for Different Vehicle Classes

\begin{tabular}{|c|c|c|}
\hline \multicolumn{3}{|c|}{ 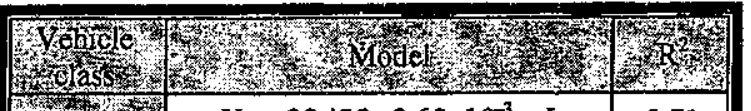 } \\
\hline U & $V_{85}=88.423+9.65 \times 10^{-3} \times L$ & 0.71 \\
\hline 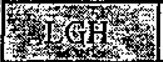 & $V_{85}=92.472+1.11 \times 10^{-2} L$ & 0.67 \\
\hline (3) & $V_{85}=81.926+5.706 \times 10^{-3} L$ & 0.35 \\
\hline
\end{tabular}

where:

$V_{85}=$ operating speed at the midpoint of tangent in $(\mathrm{km} / \mathrm{h})$; and

$L \quad=$ tangent length (m)

The best tangent models confirm that the best mathematical form of a single independent variable for different vehicle classes is the linear form of the tangent length (L). This model has a logical explanation for the effect of the independent variable (L) on the prediction of operating speed on tangents. The positive sign of the independent variable (L) means that the drivers tend to increase their speeds as (L) increases, in other words, the longer the tangent length, the higher the operating speed.

\section{Curve Models:}

Two approaches were used to estimate the operating speed models:

\section{First Approach (Direction not considered)}

The aim of this approach is to predict a single value for operating speed on a curve regardless of direction. To do this the observed speeds in both directions were merged into a single distribution, and the $V_{85 \text { th }}$ percentile computed for each curve. Therefore, the number of observations used in this approach is 10 curves. Table (6) summarizes the best curve models in this approach, as follows:

Table (6) First Approach Curve Operating Speed Models for Different Vehicle Classes

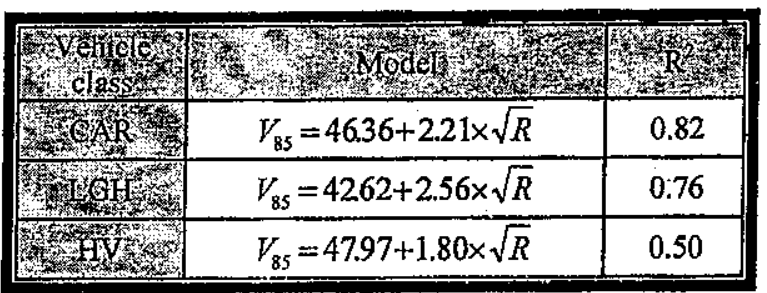


where:

$$
\begin{aligned}
& V_{8 S}=\begin{array}{l}
\text { operating speed at the midpoint of horizontal } \\
\text { curve }(\mathrm{km} / \mathrm{h}) ; \text { and }
\end{array} \\
& R=\text { curve radius (m) } \\
& \text { Second Approach (Direction considered) }
\end{aligned}
$$

\section{Second Approach (Direction considered)}

In this approach, the speed data from each direction was considered separately, creating an $85^{\text {th }}$ percentile speed for each direction in each site. The reason for using this approach is to study the effect of the direction of flow (Outer/Inner) on speed. The number of observations, in this case, is 20 curves. Table (7) summarizes the best curve models in this approach, as follows:

\begin{tabular}{|c|c|c|}
\hline \multicolumn{3}{|c|}{ 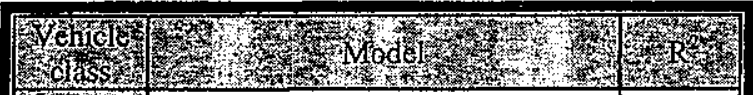 } \\
\hline & $V_{85}=4826+2.40 \times \sqrt{R}-3.80 \times D I R$ & 0.85 \\
\hline 3. & $V_{85}=4621+2.56 \times \sqrt{R}-7.20 \times D I R$ & 0.83 \\
\hline 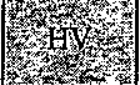 & $V_{85}=53.00+1.80 \times \sqrt{R}-1000 \times D I R$ & 0.55 \\
\hline
\end{tabular}

Table (7) Second Approach Curve Operating Speed Models for Different Vehicle Classes

where:

$V_{85}=$ operating speed midpoint of the horizontal curve at the $(\mathrm{km} / \mathrm{h})$;

$R=$ curve $\operatorname{radius}(\mathrm{m})$; and

$D I R=A$ dummy variable for direction $(D I R=0$ for inner lane and 1 for outer lane).

According to Tables (6) and (7) the best curve models, in the two approaches, showed that the best mathematical form is the root square of the curve radius $(\sqrt{R})$. The positive sign of the independent variable $(\sqrt{R})$ means that drivers tend to increase their speeds as $(R)$ increases.

In the second approach, all best curve models showed that the dummy variable DIR was significant and has a negative sign in all models. The dummy variable equals 1 for outer lanes and 0 for inner lanes. This means that vehicles traveling in the outer lanes (i.e. traveling through a larger radius) are slower than vehicles traveling in the inner lanes. While this finding may appear illogic, it could be showed that the $85^{\text {th }}$ percentile drivers are more ready to travel quickly on the inside lane than on the outside lane of a curve. As quicker vehicles are generating more sideways force, they may be more aware of the need for a lateral margin of safety on the road. On the inside of the curve this is available (providing there is no oncoming traffic) in the outer traffic lane. Drivers on the outside lane have no such margin of error and may therefore be more cautious.
Finally, it is worth mentioning that the $\mathrm{R}^{2}$ values are high in the case of curve models, reflecting the accuracy of these models to be used for prediction. In the case of tangent models, the $\mathrm{R}^{2}$ values are not as high as for curves, particularly for HV model, but are considered to be fair for modeling speeds on tangents, as found higher than those reported by Polus et al. [7].

\section{CONCLUSIONS}

From this study, the following can be concluded:

- The automatic traffic counters are capable of storing large amounts of individual vehicle data or even larger amounts of vehicle classification data.

- The analysis of variance (ANOVA) showed that there is a statistically significant difference among the observed speeds of different vehicle classes.

- No significant different was found between BUS and HGV. Theses two classes were merged into one class (HV).

- Speed distributions on each of road directions (Inner/Outer lanes), of curves, were found to be significantly different.

- Different operating speed models were developed at tangents and curves for different vehicle classes. These models are very useful and can be used for design consistency evaluations based on the operating speed methodology.

- The best tangent models confirm that the best mathematical form of a single independent variable for different vehicle classes is the linear form of the tangent length $(\mathrm{L})$.

- The best curve models showed that the best mathematical form of a single independent variable for different vehicle classes is the root square of the curve radius (R).

- The best curve models, in the second approach (direction considered), showed that the dummy variable $\mathrm{DIR}$ was significant and has a negative sign in all models. The dummy variable equals 1 for outer lanes and 0 for inner lane.

\section{REFERENCES}

[1].Hassan, Y., Sayed, T., and Tabernero, P. (2001). "Establishing Practical Approach for Design Consistency Evaluation." Journal of Transportation Engineering, ASCE, 127(4), 295302.

[2] Fitzpatrick, K., Carlson, P., Brewer, M. A., Wooldridge, M. D., and Miaou, S. (2003b). "Design Speed, Operating Speed, and Posted Speed Practices." NCHRP 504, Transportation Research Board, National Research Council, Washington, D.C. 
[3] Lamm, R., Psarianos, B., and Cafiso, S. (2002). "Safety Evaluation Process for Two-Lane Rural: A Ten Year Review." Transportation Research Record; Transportation Research Board, National Research Council, Washington, D.C., 1796, 5159.

[4] Wooldridge, M. D., Fitzpatrick, K., Harwood, D. W., Potts, I. B., Elefteriadou, L., and Torbic, D. J. (2003). "Geometric Design Consistency on HighSpeed Rural Two-Lane Roadways." NCHRP 502, Transportation Research Board, National Research Council, Washington, D.C.
[5] MetroCount. (2002). "MetroCount Traffic Executive User Manual."

[6] SPSS. (2001). "SPSS for Windows.", Rel. 11.0.0. Chicago: SPSS Inc.

[7] Polus, A., Fitzpatrick, K., and Fambro, D. (2000). "Predicting Operating Speeds on Tangent Sections of Two-Lane Rural Highways." Transportation Research Record, Transportation Research Board, National Research Council, Washington, D.C., 1737, 50-57. 